\title{
Prolonging immune responses
}

\author{
The effectiveness of cancer immunotherapies will benefit from a range of strategies - new, or borrowed from other \\ classes of therapeutic - to trigger durable immune responses.
}

$\mathrm{M}$ alignant cells and masses of malignant cells use and evolve a wide range of strategies to evade the immune system or to dampen immune activity against them. Most tellingly, cancer cells hijack mechanisms of immune tolerance (which protect from autoimmune reactions): they engage immune checkpoint 'brakes' on cytotoxic T lymphocytes to inhibit their activation, recruit regulatory immune cells that suppress or cancel out the activity of the effector T lymphocytes, downregulate proteins (such as major histocompatibility complexes) required for antigen processing and presentation, and degrade metabolically relevant amino acids and produce cytokines that suppress inflammation. Moreover, tumours can place physical barriers to lymphocyte homing and infiltration at the tumour periphery via a dysregulated vasculature with altered chemokine receptors and a dense remodelled extracellular matrix maintained by cancer-associated fibroblasts.

Many types of cancer immunotherapy - immunomodulatory molecules (such as cytokines, small-molecule agonists and adjuvants), adoptive cell therapies (typically lymphocytes with engineered cell receptors), cancer vaccines, targeted antibodies (most prominently checkpoint inhibitors) and oncolytic viruses - are designed to combat one or more of the evasion and immunosuppressive mechanisms of cancer cells and tumours. Regardless of the type of immunotherapy, its effectiveness depends on the specificity, magnitude and duration of the immune responses that it elicits. Productive antitumour immune responses need to be sustained and confined to the tumour - durable off-tumour cytotoxicity is, of course, undesirable, as is the case for cytokine storms often caused by the clinically approved chimeric antigen receptor (CAR)-T cell therapies for haematological malignancies. And robust adaptive responses are more likely to generate immunological memory. This issue of Nature Biomedical Engineering highlights a number of strategies - largely borrowed from developments in cell engineering or drug delivery - for prolonging the activity of immunomodulatory molecules and for extending the persistence of engineered effector T cells.

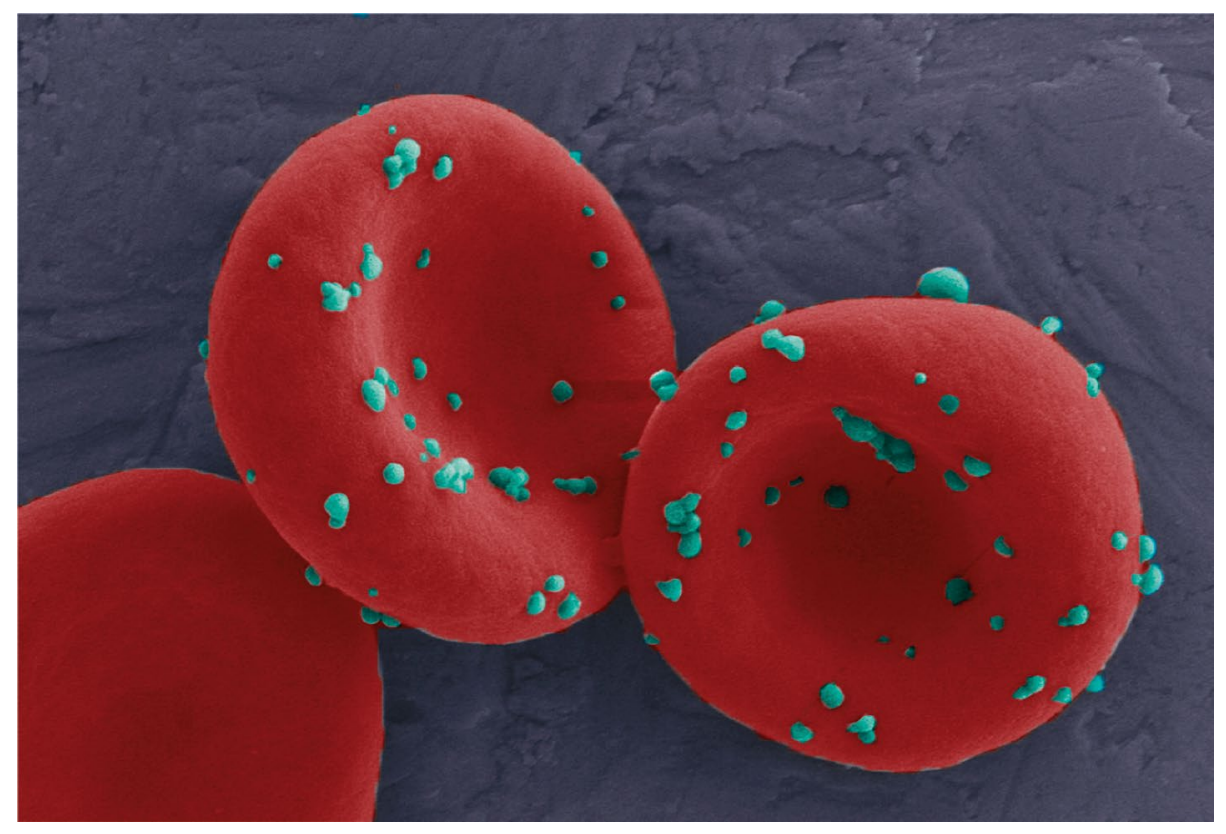

Figure reproduced with permission from the Article by Samir Mitragotri and co-authors.

A challenging aspect of the design of immunotherapies, especially for metastatic cancers, is reaching and maintaining a sufficient amount of immune-effector molecules or cells in the tumour sites (of which there can be many, and most often practically inaccessible to surgery). Yet highly perfused organs (in particular, the lungs) can be advantageously targeted via erythrocyte hitchhiking (pictured): nanoparticles non-covalently bound to red blood cells can preferentially accumulate in lung endothelium as a result of the shear forces experienced when the cells squeeze through narrow lung capillaries. Preferential nanoparticle accumulation can also be aided by coating the nanoparticles with antibodies for intercellular adhesion molecule 1 (ICAM-1; overexpressed in lung endothelium) and by the first-pass effect after cell infusion. Erythrocyte hitchhiking, which has been used to improve the blood pharmacokinetics of nanoparticle therapeutics (A. C. Anselmo et al. ACS Nano 7, 11129-11137; 2013), has now been adapted by Samir Mitragotri and colleagues to elicit immune responses against lung metastases in rodent models: in this case, the nanoparticles encapsulate the chemokine CXCL10, which attracts a range of immune-cell types. The investigators show that the preferential accumulation of CXCL10 in metastatic sites in the lungs also suppressed distant tumour growth after rechallenge, partly owing to prolonged antitumour activity from memory cytotoxic T cells.

The cytotoxic protein stimulator of interferon genes (STING) triggers interferon responses to infection and can activate antitumour pathways. However, STING agonists tested in clinical trials have shown dose-limiting toxicity and inadequate antitumour activity, partly because of their low bioavailability. Now, Jinming Gao and colleagues show that the formation of biomolecular condensates of STING via a polymer that binds multiple STING proteins prolongs STING activation and leads to more potent immune responses than those triggered by the cyclic dinucleotide cGAMP, the natural ligand of STING. They also show that the combination of cGAMP and the polymer (the two agonists bind STING on different surface sites), formulated as a nanoparticle, led to higher immune responses after intratumoral 
injection in mice bearing subcutaneous tumours and in resected cancer tissue from patients. Biomolecular condensates have been associated with amplified signalling and gene expression, and leveraging them via designer molecules to boost immune responses may turn out to be a widely applicable strategy.

For haematological malignancies, such as acute lymphoblastic leukaemia, cancer vaccines could provide a safer and long-lasting alternative to adoptive cell therapies. However, to reach sufficient potency and specificity, cancer vaccines usually require multiple immunogenic tumour-specific neoantigens. Yuhua Li, Guanghui $\mathrm{Ma}$, Wei Wei and colleagues now show that a checkpoint inhibitor (the anti-programmed-death-1 antibody) and a leukaemia-associated peptide that is highly expressed by blast cells from patients with acute myeloid leukaemia can be encapsulated in gradually degrading polymer microparticles for slow release of the antibody and antigen (over weeks in mice). With a single subcutaneous dose, the proliferation and activation of antigen-specific cytotoxic T cells was much higher than with repeated injections of free antigen, antibody and a common adjuvant. The effectiveness of other combinations of immunogens and adjuvants could also be boosted by analogous extended-release strategies designed and widely tested for small-molecule drugs and macromolecular therapeutics.
Two common drawbacks of adoptive cell therapies are that they can cause on-target off-tumour toxicity (because a majority of the tumour antigens targeted by the engineered cells are also expressed in healthy tissues), and the emergence of malignant cells resistant to the therapy (as a result of immune evasion when the targeted antigen is downregulated or mutated and not essential for tumour-cell survival). Toxicity and resistance can each be reduced by a number of strategies, but both drawbacks can be addressed by targeting neoepitopes from oncogenic driver mutations. This is what Jianzhu Chen and colleagues now show with patient-derived mouse models of acute myeloid leukaemia. The strategy prolongs therapeutic outcomes through increases in the specificity of the CAR-T cells and by limiting the development of resistance.

Adoptive cell therapies may ultimately need to be manufactured off-the-shelf from allogeneic sources to become widely accessible to patients and hopefully less costly. For this, the cells have to be engineered to avoid rejection by the host and complications from graft-versus-host disease. To generate hypoimmunogenic $\mathrm{T}$ cells, and thus prolong their survival after transplantation, Shin Kaneko and colleagues genetically engineered induced pluripotent stem cells with knocked-out genes for human leukocyte antigens and for a ligand activating natural killer cells, and with transduced genes to express an antigen that inhibits the activation of natural killer cells.
The generation of multiple edits in stem cells for the selective elimination of cellular alloimmunity is being pursued as a powerful alternative to general immunosuppression, but the generated cell products will require an extensive assessment of toxicity.

There are many other strategies to extend the immune activity elicited by immunotherapies, such as the simultaneous targeting of more than one antigen (by using bispecific T cells, for example), the ectopic expression of factors that enhance the migration of adoptive cells to tumour sites, or the modulation of the immunosuppressive tumour microenvironment by breaking down tumour stroma. And even when the transplanted cells undergo rapid cell death, this can contribute to therapeutic outcomes via mechanisms of phagocytic clearance, as Zachary Wagoner and Weian Zhao argue in a Comment also included in this issue. Because no one particular strategy is likely to work broadly across cancers, combinations of strategies bespoke, or adapted from approaches developed for other classes of therapeutic - targeting different mechanisms of immunosuppression and enhancing the survival or improving the delivery of the immune effector molecules or cells need to be extensively explored.

Published online: 17 May 2021 https://doi.org/10.1038/s41551-021-00742-9 